\title{
APLICABILIDADE DO ULTRASSOM TERAPÊUTICO EM FRATURAS COM ATRASO NA CONSOLIDAÇÃO: UMA REVISÃO INTEGRATIVA
}

\section{THE APPLICABILITY IF THERAPEUTICAL ULTRASOUND ON FRACTURES WITH CONSOLIDATION DELAYED: AN INTEGRATIVE REVIEW}

Aleques Fernandes Silva ${ }^{1}$, Joel Freires de Alencar Arrais ${ }^{1 *}$, Yago Mota Campos $^{1}$, Michelle Pereira de Lima ${ }^{1}$, Willian Cavalcante Torres Fernandes ${ }^{2}$, Rodrigo Luis Mousinho Gomes ${ }^{3}$

\footnotetext{
${ }^{1}$ Fisioterapeuta pela Fisioterapia da Faculdade de Medicina Estácio de Juazeiro do Norte (ESTÁCIO FMJ).

${ }^{2}$ Professor Especialista do curso de Fisioterapia da Faculdade de Medicina Estácio de Juazeiro do Norte (ESTÁCIO FMJ).

${ }^{3}$ Professor Mestre do curso de Fisioterapia da Faculdade de Medicina Estácio de Juazeiro do Norte (ESTÁCIO FMJ).
}

${ }^{*}$ Rua Igino Batista Maciel, s/n, em frente a Creche Zeferino Paulino de Brito - Castelo Branco, Antonina do Norte - Ceará, CEP 63570.000. Email: joel.freires00@gmail.com.

Submetido em: 19/06/2019; Aceito em: 14/03/2020.

\section{RESUMO}

O presente estudo tem como objetivo analisar na literatura se o ultrassom terapêutico possui efeito acelerador na formação do calo ósseo. Caracteriza-se como uma revisão integrativa, descritiva da literatura, que teve as seguintes bases de dados para busca dos artigos: Pubmed, Scielo e BVS, através das palavras-chave: Consolidação da fratura, Terapia por ultrassom e Modalidades de Fisioterapia, de acordo com a plataforma DeCS (Descritores em Ciências da Saúde) utilizando o operador booleano and para a junção dos mesmos. Foi encontrada vasta literatura, sendo que seguiu-se para duas etapas de seleção de acordo com os critérios de inclusão (textos gratuitos, nos idiomas inglês e português dentre os anos de 2013 e 2017 e que fossem realizados em humanos) e pelos filtros de exclusão (artigos de revisão, estudos realizados em ratos, livros e que não abordassem o tema em questão). Após análise teórica dos artigos, restaram 12 publicações incluídos nos resultados e discussão, em que verificou na maioria dos estudos que o ultrassom possui resultados positivos, acelerando o precoce de cicatrização óssea.

Palavras-Chave: Consolidação da fratura. Modalidades de fisioterapia. Terapia por ultrassom.

\section{ABSTRACT}

The present study aims to analyze in the literature whether therapeutic ultrasound has an accelerating effect on the formation of bone callus. It is characterized as an integrative, descriptive review of the literature, which had as data base for the research the journals Pubmed, Scielo and BVS, using the keywords: Fracture 
Healing, Ultrasonic Therapy and Physical Therapy Modalities, according to the DeCS platform (Health Sciences Descriptors) using the boolean operator and to join them. A vast literature was found of which they went through two stages of selection according to the inclusion criterium (free text, in English and Portuguese between the years 2013 and 2017 and that were carried out in humans) and through the exclusion filters (articles review, studies carried out on rats, books that did not address the topic in question). After screening, 12 articles remained to be analyzed, which were included in this study. With the discussion of the selected articles it is possible to observe that ultrasound in most studies has a positive result, accelerating the bone healing process.

Keywords: Fracture Healing. Physical Therapy Modalities. Ultrasonic Therapy.

\section{INTRODUÇÃO}

As fraturas caracterizam-se como a descontinuidade óssea. Se manifesta a partir de movimentos ósseos no local fraturado, dor e fraqueza no membro atingido (RUARO, 2004; OMS, 2011; NOZAKA et al., 2014a). Para considerar uma fratura cicatrizada, ela deve atender a critérios clínicos e radiológicos: não apresentar movimento ou crepitação local, livre de dor e ao mínimo de três a quatro córtices de pontes visualizadas em radiografia (ZURA et al., 2015a).

Pseudoartrose é uma complicação na qual uma fratura não consolidou e/ou não apresenta sinais clínicos ou radiológicos de calos moles. Esta complicação repercute com dor, deformidades ósseas, compressão neurológica e necrose óssea. Não se pode definir especificamente o tempo da não união óssea, geralmente são diagnosticadas com nove meses ou mais sem início de consolidação (GAIARSA et al., 2011; POVLSEN; POVLSEN, 2015; OLIVEIRA et al., 2017).

De acordo com Ferreira et al. (2009) existem fatores que podem contribuir com a não consolidação óssea, como a instabilidade da fratura e suprimento sanguíneo falho no local fraturado. Para fraturas com atraso na consolidação, um dos possíveis tratamentos não invasivos é o ultrassom terapêutico (UST), pois estimulam as temperaturas metabólicas locais intensificando o processo de consolidação óssea (NOLTE et al., 2001; PEREIRA-FONTES et al., 2013).

Diversos estudos afirmam que o UST é um bom tratamento não invasivo para a reparação dos tecidos (OLSSON et al., 2008). Trata-se de um dispositivo de baixa intensidade que acarreta aceleração normal do reparo das fraturas e o tempo é significativamente menor quando comparado as fraturas de um grupo placebo (HECKMAN et al., 1994; KRISTIANSEN et al., 1997). E está associado a altas taxas de curas (ZURA et al., 2015a), união completa de fraturas (POVLSEN; POVLSEN, 2015) e redução da dor das repercussões de tal lesão (NOLTE et al., 2016).

Sabendo que as fraturas são casos que o fisioterapeuta encontra frequentemente em sua prática clínica, julga-se de inteira importância que o mesmo possua o conhecimento de métodos de tratamento que possam acelerar a recuperação dessas lesões que trazem déficit de funcionalidade e podem apresentar quadros com diversas complicações. 
Com isso, o estudo tem como objetivo analisar na literatura se o UST possui efeito acelerador de formação do calo ósseo em fraturas com atraso na consolidação. Necessitando a investigação da aplicabilidade no tratamento de fraturas com atraso na consolidação, se são benéficos ou não na melhora das fraturas e quais os parâmetros mais utilizados na literatura.

\section{MATERIAL E MÉTODOS}

A metodologia de pesquisa caracteriza-se por uma revisão integrativa da literatura que analisa os efeitos da aplicabilidade do ultrassom terapêutico no tratamento das fraturas com atraso de consolidação. Foram utilizados para embasamento teórico as publicações encontradas no período de 01/03/2018 à 20/03/2018.

O procedimento para a coleta dos artigos científicos foi realizado utilizando as bases de dados: Pubmed, Scielo e BVS através do endereço eletrônico. Foram utilizados os seguintes descritores de acordo com a plataforma DeCS (Descritores em ciências e saúde): Ultrasonic Therapy, Fracture Healing e Physical Therapy Modalities utilizando para a combinação desses descritores o operador boleano and.

Nas bases de dados Scielo, Pubmed e BVS encontrou-se 8.133 publicações. Foram selecionados por disponibilidade do texto completo gratuitamente, idiomas inglês e português dentre os anos de 2013 à 2017, estudos realizados em humanos e assunto principal consolidação da fratura. Dos quais perduraram 221 artigos para a leitura. Cujo após a leitura do título e resumo, 25 se tratavam de revisões ou meta-análises, 7 se repetiam entre as bases, 1 não compreendia a população desejada, foi encontrado um capítulo de livro, 2 artigos não se obtiveram acesso à leitura, 173 não englobavam o tema restando 12 trabalhos para embasamento teórico.

Figura 1.

O delineamento do estudo está descrito mais detalhado e resumido na

Figura 1 - Busca nas bases de dados.

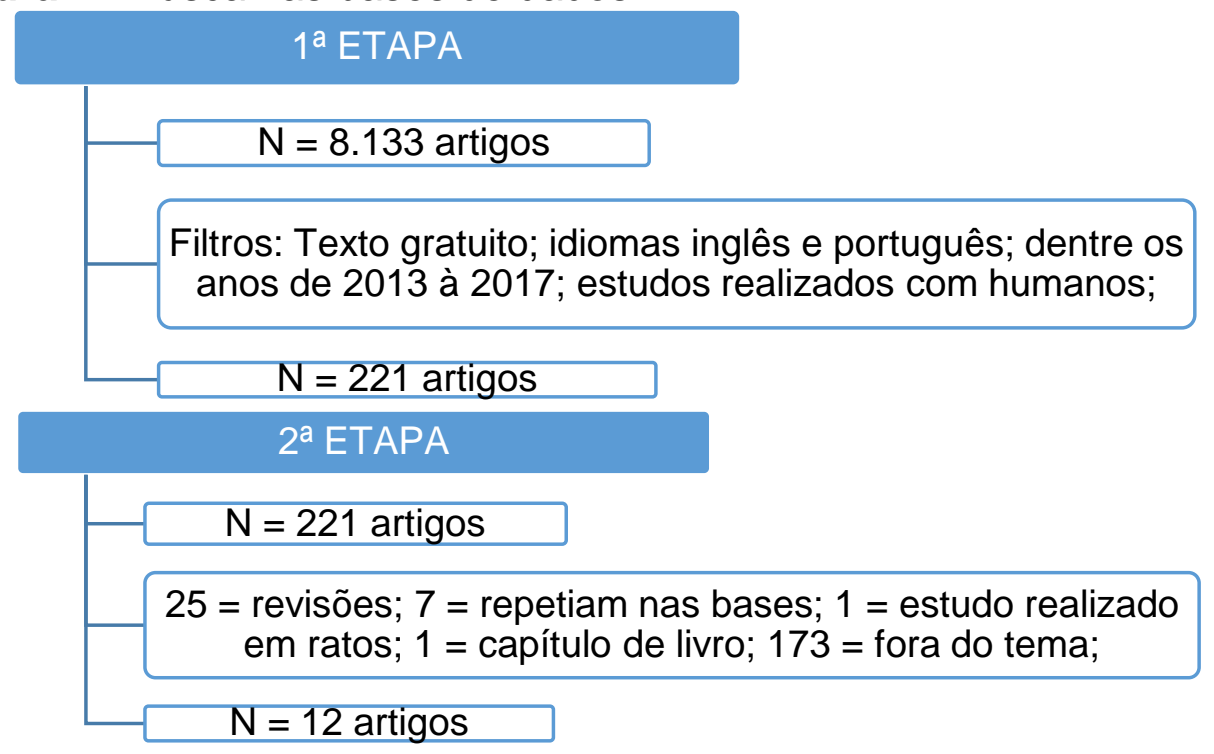

Fonte: os autores. 
Por tratar-se de uma revisão integrativa da literatura, o presente trabalho assegura-se por meio da Resolução 510/16 do Conselho Nacional de Saúde, justificando assim que o mesmo, não necessitou ser submetido ao Comitê de Ética em Saúde.

\section{RESULTADOS E DISCUSSÃO}

De acordo com o levantamento literário foram encontrados os seguintes estudos os quais apresentam os métodos de tratamentos aplicados e resultados alcançados no Quadro 1.

No Quadro 1 observa-se que a maioria dos estudos apresentam resultados positivos na utilização do ultrassom pulsado de baixa intensidade nas fraturas com atraso na consolidação óssea. De acordo com BIGLARI et al. (2016) não se sabe ao certo se a utilização do ultrassom tem relevância no tratamento de não-uniões, entretanto em outros estudos, o uso do ultrassom está associado com elevadas taxas de cura (ZURA et al., 2015a), consolidação completa de fraturas (POVLSEN; POVLSEN, 2015) e diminuição da dor (NOLTE et al., 2016). Para o tratamento com ultrassom dessa complicação necessita-se que a fratura esteja estabilizada (NOZAKA et al., 2014b)

Zuara et al. (2015a) definem alguns critérios de inclusão para análise de cura das fraturas que consistiam, data em que ocorreu a fratura, início do uso do ultrassom, encerramento do tratamento e os resultados que diante da aplicação do ultrassom apresentou cicatrização, a fratura deveria atender os critérios clínicos e radiológicos; não apresentar movimento ou crepitação no local fraturado, livre de dor por estresse manual e pelo menos de três a quatro pontes de calo nas radiografias. Nolte et al. (2016), Zuara et al. (2015b) realizaram seus estudos com base nos mesmos critérios. Contudo, para Salem e Schmelz (2014) a cicatrização foi estipulada com o tempo, respeitando três fases: fase latente, fase de distração e fase de consolidação ou maturação.

Nos estudos de Busse et al. (2014) e Biglari et al. (2016), a avaliação da consolidação das fraturas ocorreu em várias ocasiões, de semanas a meses após o fim do tratamento. No momento da alta, na sexta semana, e posteriormente ao terceiro mês foram realizadas mais 5 avaliações, sendo a última no décimo segundo mês do pós-operatório (BUSSE et al., 2014).

Em alternativa, os pacientes foram acompanhados na unidade ambulatorial foram examinados radiologicamente e clinicamente na sexta e décima segunda semana, com 4 avaliações sendo a última também no décimo segundo mês, ou até ocorrer a consolidação óssea (BIGLARI et al., 2016). Por outro lado, para Liu et al. (2014), as condições de cicatrização óssea foram determinadas quando os pacientes não especificam processo álgico, caso contrário a imobilização continuaria e com uma semana seria realizado uma reavaliação.

Os resultados adquiridos nos estudos de Busse et al. (2016) e Biglari et al. (2016), apontam que não há relevância entre o grupo com ultrassom versus o grupo controle, os quais foram avaliados durante o processo de cura radiológica ou até um ano após a fixação intramedular, e que em apenas 20 casos $(32,8 \%)$ o tratamento foi bem-sucedido, sendo necessária realização de cirurgia adicional em $62,2 \%$ dos casos. 
Quadro 1 - Artigos que abordam os resultados da aplicação do UST em fraturas com atraso na consolidação.

\begin{tabular}{|c|c|c|c|c|}
\hline AUTOR & TÍTULO & TIPO & MÉTODOS & RESULTADOS \\
\hline $\begin{array}{l}\text { NOZAKA et } \\
\text { al., 2014a. }\end{array}$ & $\begin{array}{l}\text { Combined effect of } \\
\text { teriparatide and low- } \\
\text { intensity pulsed } \\
\text { ultrasound for nonuion: } \\
\text { a case report. }\end{array}$ & $\begin{array}{l}\text { Relato de } \\
\text { caso. }\end{array}$ & $\begin{array}{l}\text { Caso de um homem de } 56 \text { anos, fratura do } \\
\text { eixo femoral submetido a osteossintese } \\
\text { intramedular. Onde não apresentou sinal de } \\
\text { consolidação após } 3 \text { meses. Iniciou-se a } \\
\text { aplicação do ultrassom pulsado de baixa } \\
\text { intensidade, após } 3 \text { meses iniciado } \\
\text { tratamento com hormônio paratireóide em } \\
\text { combinação com o ultrassom. }\end{array}$ & $\begin{array}{l}\text { Com o uso somente do ultrassom, apresentou- } \\
\text { se após três meses sinais de consolidação } \\
\text { leve. Com o uso da terapia combinada do } \\
\text { ultrassom com hormônio paratireoide houve } \\
\text { cura completa após } 6 \text { meses. }\end{array}$ \\
\hline $\begin{array}{l}\text { NOZAKA et } \\
\text { al., 2014b. }\end{array}$ & $\begin{array}{l}\text { Pathological fracture of } \\
\text { the femur in alagille } \\
\text { syndrome that was } \\
\text { treated with low- } \\
\text { intensity pulsed } \\
\text { ultrasound stimulation } \\
\text { and an llizarov ring } \\
\text { fixator: a case report. }\end{array}$ & $\begin{array}{l}\text { Relato de } \\
\text { caso. }\end{array}$ & $\begin{array}{l}\text { Menina de } 9 \text { anos com síndrome de Alagille, } \\
\text { apresentou fratura patológica do eixo femoral } \\
\text { direito, tratada com tração esquelética, com } \\
\text { reposicionamento difícil. Decidiu-se usar } \\
\text { redução direta com fixador de anel llizarov. } \\
\text { Imediatamente após a cirurgia iniciou a } \\
\text { estimulação ultrassônica de baixa } \\
\text { intensidade durante } 20 \text { min, F de } 1,5 \mathrm{MHz} \text {, } \\
\text { Lp } 200 \mathrm{~ms} \text {, F de repetição de sinal de } 1 \mathrm{kHz} \\
\text { e intensidade de } 30 \mathrm{~mW} / \mathrm{CM}^{2} \text {, e terapia física } \\
\text { envolvendo andar com peso na perna } \\
\text { operada. }\end{array}$ & $\begin{array}{l}\text { O uso do ultrassom foi continuado, e a } \\
\text { paciente foi autorizado a andar sem muletas. } \\
\text { As radiografias mostraram cicatrização da } \\
\text { fratura aos } 53 \text { dias pós-operatório. } 74 \text { dias no } \\
\text { pós-operatório, o quadro foi removido e a } \\
\text { paciente se recuperou anatomicamente e } \\
\text { funcionalmente. }\end{array}$ \\
\hline $\begin{array}{l}\text { BUSSE et } \\
\text { al., } 2014 .\end{array}$ & $\begin{array}{l}\text { Trial to re-evaluate } \\
\text { ultrasound in the } \\
\text { treatment of tibial } \\
\text { fractures (TRUST): a } \\
\text { multicenter } \\
\text { randomized pilot } \\
\text { study. }\end{array}$ & $\begin{array}{c}\text { Estudo } \\
\text { Randomizado } \\
\text { Multicêntrico. }\end{array}$ & $\begin{array}{l}51 \text { pacientes, } 37 \text { foram fraturas fechadas e } \\
14 \text { fraturas abertas. Após uma fixação com } \\
\text { uma haste intramedular, os pacientes foram } \\
\text { alocados para o grupo com ultrassom } \\
\text { pulsado de baixa intensidade ou um } \\
\text { dispositivo desativado. Os grupos realizaram } \\
\text { auto tratamento por } 20 \text { minutos. }\end{array}$ & $\begin{array}{l}\text { As medidas repetidas de análise de variância } \\
\text { descobriram que o tratamento com ultrassom } \\
\text { pulsado de baixa intensidade } x \text { terapia } \\
\text { simulada não foi significantemente associado } \\
\text { à melhora nas pontuações PCS SF-36 (tempo } \\
x \text { interação do tratamento. } p=0,27 \text { ), } \\
\text { pontuações HUI-III (tempo } x \text { interação do } \\
\text { tratamento, } p=0,31 \text { ), ou pontuação RUST } \\
\text { (tempo } x \text { interação de tratamento, } p=053 \text { ). } O \\
\text { paciente demonstro resultados piores nas três } \\
\text { medidas. }\end{array}$ \\
\hline
\end{tabular}


Quadro 1, cont

\begin{tabular}{|c|c|c|c|c|}
\hline $\begin{array}{l}\text { LIU et al., } \\
2014 .\end{array}$ & $\begin{array}{l}\text { Ultrasound treatment } \\
\text { for accelerating } \\
\text { fracture healing of the } \\
\text { distal radius. A control } \\
\text { study. }\end{array}$ & $\begin{array}{l}\text { Estudo piloto } \\
\text { randomizado. }\end{array}$ & $\begin{array}{l}81 \text { pacientes, foram reduzidos e } \\
\text { imobilizados. Foram divididos em dois } \\
\text { grupos: G1 (ultrassom = 41) G2 (controle = } \\
40 \text { ). O tratamento com ultrassom; } 15 \mathrm{~min} \\
\text { diários intensidade de } 30 \mathrm{~mW} / \mathrm{cm}^{2}, \\
\text { frequência de } 1,5 \mathrm{MHz} \text { e duração de pulso } \\
\text { de } 200 \text { us. }\end{array}$ & $\begin{array}{l}\text { O tempo médio para a cicatrização clínica foi } \\
\text { de } 32,04 \pm 2,58 \text { dias no grupo de ultrassom e } \\
40,75 \pm 5,12 \text { dias no grupo controle. O tempo } \\
\text { de cicatrização clínica no grupo de ultrassom } \\
\text { foi menor do que no grupo controle. }\end{array}$ \\
\hline $\begin{array}{l}\text { SALEM; } \\
\text { SCHMELZ, } \\
2014 .\end{array}$ & $\begin{array}{l}\text { Low-intensity pulsed } \\
\text { ultrasound shortens } \\
\text { the treatment time in } \\
\text { tibial distraction } \\
\text { osteogenesis. }\end{array}$ & $\begin{array}{l}\text { Ensaio } \\
\text { controlado } \\
\text { randomizado. }\end{array}$ & $\begin{array}{l}21 \text { pacientes divididos em um grupo } \\
\text { experimental } 12 \text { pessoas (distração do calo } \\
\text { foi apoiada com estimulação com US) e } \\
\text { grupo controle } 9 \text { pacientes (não foram } \\
\text { submetidos ao US). Em todos os casos } \\
\text { fizeram o uso de fixadores de anel llizarov. A } \\
\text { terapia por ultrassom utilizou os valores: F } \\
1,5 \mathrm{MHz} \text {, Forma: pulsado, Lp } 200 \mu \mathrm{s}, \mathrm{F} \text { de } \\
\text { repetição do sinal } 1 \mathrm{kHz} \text { e Intensidade de } 30 \\
\mathrm{~mW} / \mathrm{cm}^{2} \text { por } 20 \text { minutos diários. }\end{array}$ & $\begin{array}{l}\text { Na fase de distração o aumento diário na } \\
\text { densidade óssea significou uma melhoria em } \\
14 \% \text { no grupo experimental. Na fase de } \\
\text { consolidação esse aumento equivaleu em } \\
58 \% \text { no grupo experimental. Quanto ao tempo } \\
\text { de cicatrização, o grupo experimental } \\
\text { necessitou de uma média de } 33 \text { dias, } \\
\text { enquanto o grupo controle uma média de } 45 \\
\text { dias para alcançar o mesmo objetivo. }\end{array}$ \\
\hline $\begin{array}{l}\text { ZURA et } \\
\text { al., } 2015 a .\end{array}$ & $\begin{array}{l}\text { A cohort study of } \\
4,190 \text { patients treated } \\
\text { with low-intensity } \\
\text { pulsed ultrasound } \\
\text { (lipus): findings in the } \\
\text { elderly versus all } \\
\text { patients. }\end{array}$ & $\begin{array}{l}\text { Estudo de } \\
\text { corte. }\end{array}$ & $\begin{array}{l}4.190 \text { registros de fraturas que foram } \\
\text { tratadas com ultrassom pulsátil de baixa } \\
\text { intensidade. }\end{array}$ & $\begin{array}{l}\text { Nos } 4.190 \text { pacientes com fratura recente, a } \\
\text { taxa de cura da fratura foi de } 96,2 \% \text {. O tempo } \\
\text { de tratamento foi significativamente curto entre } \\
\text { os pacientes que curaram. As pessoas acima } \\
\text { de } 60 \text { anos tratadas com ultrassom } \\
\text { apresentam taxas de cura semelhantes a } \\
\text { população como um todo. }\end{array}$ \\
\hline $\begin{array}{l}\text { FARKASH } \\
\text { et al., } 2015 .\end{array}$ & $\begin{array}{l}\text { Low-intensity pulsed } \\
\text { ultrasound for treating } \\
\text { delayed union } \\
\text { scaphoid fractures: } \\
\text { case series. }\end{array}$ & $\begin{array}{l}\text { Série de } \\
\text { casos. }\end{array}$ & $\begin{array}{l}29 \text { pacientes do sexo masculino com fratura } \\
\text { de escafoide. } 13 \text { pacientes diagnosticados } \\
\text { precocemente; foram tratados com } \\
\text { imobilização e } 3 \text { meses iniciaram a terapia } \\
\text { com ultrassom. } 16 \text { pacientes diagnosticados } \\
\text { tardiamente; tratados por gesso e ultrassom } \\
\text { no momento do diagnóstico. Doses diárias } \\
\text { de } 20 \text { min, baixa intensidade de } 30 \mathrm{~mW} / \mathrm{cm}^{2} \text {, } \\
\text { F de } 1,5 \mathrm{MHz} \text { e } \mathrm{Lp} \mathrm{de} 200 \mu \mathrm{s} \text {, taxa de } \\
\text { repetição de } 1,0 \mathrm{KHz} \text {. }\end{array}$ & $\begin{array}{l}\text { No total, } 22 \text { de } 29(76 \%) \text { das fraturas foram } \\
\text { curadas. } 12 \text { de } 13(92 \%) \text { do grupo com } \\
\text { diagnóstico precoce e } 10 \text { de } 16 \text { (63\%) do } \\
\text { grupo com diagnóstico tardio. }\end{array}$ \\
\hline
\end{tabular}


Quadro 1, cont

\begin{tabular}{|c|c|c|c|c|}
\hline $\begin{array}{l}\text { ZURA et } \\
\text { al., 2015b. }\end{array}$ & $\begin{array}{l}\text { Treatment of chronic } \\
(>1 \text { year) fracture } \\
\text { nonunion: Heal rate in } \\
\text { a cohort of } 767 \\
\text { patients treated with } \\
\text { low-intensity pulsed } \\
\text { ultrasound (LIPUS). }\end{array}$ & $\begin{array}{l}\text { Estudo de } \\
\text { coorte } \\
\text { retrospectivo. }\end{array}$ & $\begin{array}{l}\text { O estudo coletou os resultados de um } \\
\text { registro prospectivo de pacientes coletados } \\
\text { ao longo de } 4 \text { anos. Um total de } 7.884 \\
\text { fraturas; } 4.190 \text { agudas, } 2927 \text { não-união } \\
\text { tardia, } 767 \text { não-uniões crônicas. }\end{array}$ & $\begin{array}{l}\text { O tratamento com ultrassom pulsado de baixa } \\
\text { intensidade foi associado a uma alta taxa de } \\
\text { cura ( } 86,2 \%) \text { em uma coorte de registro de } \\
767 \text { fraturas de não-união que não atingiram a } \\
\text { consolidação dentro do intervalo de um ano. }\end{array}$ \\
\hline $\begin{array}{l}\text { POVLSEN; } \\
\text { POVLSEN, } \\
2015 .\end{array}$ & $\begin{array}{l}\text { Low-intensity pulsed } \\
\text { ultrasound treatment } \\
\text { as an alternative to } \\
\text { vascular bone graft } \\
\text { surgery for a 5-year- } \\
\text { long ulnar non-union in } \\
\text { a patient with } \\
\text { haemochromatosis. }\end{array}$ & $\begin{array}{l}\text { Relato de } \\
\text { caso. }\end{array}$ & $\begin{array}{l}\text { Fratura de Colles direita diagnosticada a } \\
\text { mais de } 30 \text { anos, realizou nesse período } 4 \\
\text { cirurgias resultando danos nos tecidos moles } \\
\text { e ósseo. Após todas as tentativas paciente } \\
\text { escolheu a forma conservadora (ultrassom } \\
\text { pulsado de baixa intensidade; } 20 \text { min diários } \\
\text { por } 4 \text { meses). } \mathrm{F} \text { de } 1,5 \mathrm{MHz}, \mathrm{P} \text { de } 117 \mathrm{~mW} \\
\text { em uma área de } 3,88 \mathrm{~cm}^{2} .\end{array}$ & $\begin{array}{l}\text { A união completa foi acompanhada por } \\
\text { diminuição na dor do antebraço. }\end{array}$ \\
\hline $\begin{array}{l}\text { BIGLARI et } \\
\text { al., } 2016 .\end{array}$ & $\begin{array}{l}\text { Failed treatment of } \\
\text { long bone nonunions } \\
\text { with low intensity } \\
\text { pulsed ultrasound. }\end{array}$ & $\begin{array}{c}\text { Estudo } \\
\text { observacional. }\end{array}$ & $\begin{array}{l}61 \text { casos }(91,8 \% \text { homens, idade entre } 18 \text { e } \\
63 \text { anos) tratados por } 20 \text { minutos, com F de } \\
1,5 \mathrm{MHz}( \pm 5 \%), \mathrm{P} \text { de } 30 \mathrm{~mW} / \mathrm{cm}^{2}( \pm 30 \%), \mathrm{DP} \\
\text { de } 200 \text { Is }( \pm 10 \%) \text { e repetição do sinal de } 1 \\
\mathrm{kHz}( \pm 10 \%) . \text { Tratamento na extremidade } \\
\text { inferior: tíbia }(57,4 \%) \text { e fêmur }(18,0 \%) \text {. }\end{array}$ & $\begin{array}{l}\text { Em } 32,8 \% \text { o tratamento foi bem-sucedido e } \\
62,2 \% \text { realizaram cirurgia adicional. Após a } \\
\text { conclusão da terapia, } 5 \text { pacientes }(8,2 \%) \\
\text { relataram melhora da dor, } 13(21,3 \%) \text { melhora } \\
\text { mínima, } 37(60,7 \%) \text { sem melhorar e } 1 \text { paciente } \\
(1,6 \%) \text { disse que a dor se tornou mais grave. }\end{array}$ \\
\hline $\begin{array}{l}\text { NOLTE et } \\
\text { al., 2016. }\end{array}$ & $\begin{array}{l}\text { Heal rate of metatarsal } \\
\text { fractures: a propensity- } \\
\text { matching study of } \\
\text { patients treated with } \\
\text { low-intensity pulsed } \\
\text { US (lipus) vs. Surgical } \\
\text { and other treatments. }\end{array}$ & $\begin{array}{l}\text { Estudo de } \\
\text { corte } \\
\text { retrospectivo } \\
\text { observacional. }\end{array}$ & $\begin{array}{l}\text { Um total de } 594 \text { casos com fraturas } \\
\text { metatarsiana, dos quais } 368 \text { apresentaram } \\
\text { fratura fresca e } 226 \text { apresentaram fratura } \\
\text { retardada. } 40,2 \% \text { das fraturas a localização } \\
\text { era desconhecida. Aproximadamente } 63,5 \% \\
\text { se tratava de fraturas fechadas. }\end{array}$ & $\begin{array}{l}\text { A taxa de cura com foi de } 97,3 \% \text {, para os } \\
\text { pacientes que receberam o LIPUS, } \\
\text { comparável à taxa de cura de } 95,3 \% \text { entre os } \\
\text { pacientes atendidos em } 2011 \text { que não } \\
\text { receberam o tratamento. }\end{array}$ \\
\hline $\begin{array}{l}\text { BUSSE et } \\
\text { al., } 2016 .\end{array}$ & $\begin{array}{l}\text { Re-evaluation of low } \\
\text { intensity pulsed US in } \\
\text { treatment of tibial } \\
\text { fractures (TRUST): } \\
\text { randomized clinical } \\
\text { trial. }\end{array}$ & $\begin{array}{l}\text { Ensaio clínico } \\
\text { multicêntrico, } \\
\text { randomizado } \\
\text { cego. }\end{array}$ & $\begin{array}{l}\text { De } 3.105 \text { pacientes foram selecionados } 501 \text {, } \\
\text { alocados para autoadministrar o US pulsado } \\
\text { de baixa intensidade diário }(n=250) \text { ou usar } \\
\text { um dispositivo simulador }(n=251) \text { até a } \\
\text { fratura apresentar cura radiográfica ou até } \\
\text { um ano após a fixação intramedular. }\end{array}$ & $\begin{array}{l}\text { Os resultados não mostram impacto nos } \\
\text { escores entre ultrassom pulsado de baixa } \\
\text { intensidade e grupo controle em relação ao } \\
\text { tempo de tratamento ou outras medidas } \\
\text { funcionais. }\end{array}$ \\
\hline
\end{tabular}

Fonte: os autores

um ano após a fixação intramedular. 
O que difere de outros resultados encontrados que apontam que o tempo de cicatrização em comparação com o grupo controle foi menor em média de $32,04 \pm 2,58$ dias (LIU et al., 2014), que houve aumento na densidade óssea observada em duas fases a qual a primeira representou $14 \%$ de melhora e na segunda um aumento de 58\% no grupo experimental, com redução do tempo de cicatrização em média apenas 33 (SALEM; SCHMELZ, 2014).

Zura et al. (2015a) e Nolte et al. (2016), relatam também que o uso do ultrassom foi responsável por uma taxa de cura de 96,2\% e 97,3\% respectivamente com redução no tempo de tratamento, e verificou-se que a população idosa teria um benefício maior pois as taxas de cura se asemelham a taxa de cura ao restante da população, apresentando altas porcentagens de cura, que é reafirmado por Farkash et al. (2015), que diz, que cerca de $76 \%$ das fraturas tratadas foram curadas, $92 \%$ relacionadas a fraturas com diagnóstico precoce e $63 \%$ com diagnóstico tardio, com isso certifica-se que os resultados apresentam melhores efeitos com um diagnóstico precoce.

Alguns estudos apresentaram efetividade com o tratamento do ultrassom associado ao uso do hormônio paratireoide, a qual houve consolidação após o sexto mês de tratamento (NOZAKA et al., 2014a), ou terapia física composta por caminhada com dissociação com peso na perna operada (descarga de peso), sendo que, o processo de consolidação foi observado após 53 dias do pósoperatório (NOZAKA et al., 2014b).

Para Busse et al. (2014) evidenciaram que o tratamento não foi significativo, observaram também eventos adversos no estudo: casos de infecção de feridas remoção dos fixadores por desconforto e infecção profunda, desbridamentos por infecção, casos de pneumonias, trombose venosa profunda e embolia pulmonar, corroborando com o estudo de Biglari et al. (2016) que relacionaram que o ultrassom não deve ser utilizado quando a lesão apresenta sinais de infecção ativa.

Analisando as literaturas as quais apresentam resultados positivos nas fraturas com atraso na consolidação, há alguns parâmetros que os artigos apresentam igualdade em suas formas de tratamento: Frequência de 1,5 MHz, Largura de pulso de $200 \mathrm{mn}$ e Frequência de repetição de $1 \mathrm{KHz}$ (LIU et al., 2014; NOZAKA et al., 2014b; SALEM; SCHMELZ, 2014; FARKASH et al., 2015; POVLSEN; POVLSEN, 2015). Divergindo de alguns artigos que em sua maioria apresentaram tempo de 20 minutos, um deles utilizou apenas 15 minutos de aplicação diária (LIU et al., 2014), outro parâmetro utilizado pela maioria de 30 $\mathrm{mW} / \mathrm{cm}^{2}$, apenas um estudo fugiu dessa aplicação e utilizou $117 \mathrm{~mW} / \mathrm{cm}^{2}$ (POVLSEN; POVLSEN, 2015).

\section{CONCLUSÃO}

Dentre os estudos abordando o tratamento com UST em fraturas com atraso da consolidação discutidos na revisão apresentou-se com resultados positivos, podendo considerar como medida de tratamento conservador, bem como no pós-operatório recente ou tardio.

Porém, foi verificada uma carência de estudos científicos voltados a participação de profissionais de Fisioterapia para aprimorar os conhecimentos e prática de um recurso incluso no arsenal. Desta forma, faz-se necessário o 
interesse por parte destes profissionais fisioterapeutas a busca de mais pesquisas envolvendo a temática, corroborando assim com os achados da pesquisa.

\section{REFERÊNCIAS}

BIGLARI, B. et al. Failed treatment of long bone nonunions with low intensity pulsed ultrasound. Archives of Orthopaedic and Trauma Surgery, v. 136, p. 1121-1134, 2016.

BUSSE, W. J. et al. Re-evaluation of low intensity pulsed ultrasound in treatment of tibial fractures (TRUST): randomized clinical trial. British Medical Journal, 2016.

BUSSE, W. J. et al. Trial to re-evaluate ultrasound in the treatment of tibial fractures (TRUST): a multicenter randomized pilot study. Trials, v. 15, n. 206, 2014.

FARKASH, U. et al. Low-intensity pulsed ultrasound for treating delayed union scaphoid fractures: case series. Journal of Orthopaedic Sugery and Research, v. 10, n. 72, 2015.

FERREIRA, L. M. et al. Modelo experimental em ratos para o desenvolvimento de pseudoartrose. Revista do Colégio Brasileiro de Cirurgiões, v. 36, n. 6, p. 514-518, 2009.

GAIARSA, P. G.; REIS, R. P.; TARGA, C. H. W. Pseudoartrose. IN: POZZI, I. et al., (Coord). Manual de Trauma Ortopédico. São Paulo: SBOT - Sociedade Brasileira de Ortopedia e Traumatologia, 2011: p. 239-243.

HECKMAN, J. D. et al. Acceleration of tibial fracture-healing by non-invasive, lowintensity pulsed ultrasound. Journal of Bone \& Joint Surgery, v. 76, n. 1, p. 2634, 1994.

KRISTIANSEN, T. K. et al. Accelerated healing of distal radial fractures with the use of specific, low-intensity ultrasound. A multicenter, prospective, randomized, double-blind, placebo-controlled study. Journal of Bone \& Joint Surgery, v. 79, n. 7, p. 961-973, 1997.

LIU, Y. et al. Ultrasound treatment for accelerating fracture healing of the distal radius. A control study. Acta Cirúrgica Brasileira, v. 29, n. 11, p. 765-770, 2014.

NOLTE, P. et al. Heal rate of metatarsal fractures: a propensity-matching study of patients treated with low-intensity pulsed ultrasound (lipus) vs. Surgical and other treatments. Injury, International Journal of the Care of the Injured, v. 47, n. 11, p. 2584-2590, 2016. 
NOLTE, P. A. et al. Low-intensity pulsed ultrasound in the treatment of nonunions. The Journal of Trauma, v. 51, n. 4, p. 693-702, 2001.

NOZAKA, K. et al. Combined effect of teriparatide and low-intensity pulsed ultrasound for nonunion: a case report. BMC Research Notes, v. 317, n. 7, 2014a.

NOZAKA, K. et al. Pathological fracture of the femur in alagille syndrome that was treated with low-intensity pulsed ultrasound stimulation and an llizarov ring fixator: a case report. BMC Musculoskeletal Disorders, v. 15, n. 225, 2014b.

OLIVEIRA, G. R. O. et al. O uso de proteínas morfogenéticas ósseas (BMP) e pseudoartroses, uma revisão de literatura. Revista Brasileira de Ortopedia, v. 52, n. 2, p. 124-140, 2017.

OLSSON, C. D. et al. Ultra-som terapêutico na cicatrização tecidual. Ciência Rural, v. 38, n. 4, p. 1199-1207, 2008.

OMS. Relatório Mundial sobre a Deficência. Lexicus Serviços Lingüísticos.São Paulo: SEDPcD, 2011.

PEREIRA-FONTES, J. A. et al. Efeito do ultrassom terapêutico de baixa intensidade em fratura induzida em tíbia de ratos. Acta Ortopédica Brasileira, v. 21, n. 1 , p. 18-22, 2013.

POVLSEN, D. S.; POVLSEN, B. Low-intensity pulsed ultrasound treatment as an alternative to vascular bone graft surgery for a 5-year-long ulnar non-union in a patient with haemochromatosis. British Medical Journal, v. 16, n. 5, 2015.

RUARO, A. F. Ortopedia e Traumatologia: Temas Fundamentais e a Reabilitação. 1. ed. - Umuarama: Elenco, 2004.

SALEM, H. K.; SCHMELZ, A. Low-intensity pulsed ultrasound shortens the treatment time in tibial distraction osteogenesis. International Orthopaedics, $v$. 38, n. 7, p. 1477-1482, 2014.

ZURA, R. et al. A cohort study of 4,190 patients treated with low-intensity pulsed ultrasound (lipus): findings in the elderly versus all patients. BMC Musculoskeletal Disorders, v. 16, n. 45, $2015 a$.

ZURA, R. et al. Treatment of chronic (>1 year) fracture nonunion: heal rate in a cohort of 767 patients treated with low-intensity pulsed ultrasound (lipus). Injury International Journal of the Care of the Injured, v. 46, n. 10, p. 2036-2041, 2015b. 